\title{
Inverse raytracing based on Monte-Carlo radiative transfer simulations
}

\author{
S. Wolf* \\ Thüringer Landessternwarte Tautenburg, Sternwarte 5, 07778 Tautenburg, Germany \\ Received 4 November 1999 / Accepted 6 September 2001

\begin{abstract}
A method for the detailed investigation of polarization maps and images obtained from high-resolution observations is described in this paper. It has been developed for the analysis of the environment of young stellar objects (YSO), ultra-compact HII regions (UCHII), and the optically thin light scattering region of active galactic nuclei (AGN). The method is based on a 3D Monte-Carlo radiative transfer (RT) code. The main principle of this method lies in the detailed investigation of the paths of test-photons in the considered medium. To demonstrate the efficiency and limit of this method, it is applied to a simple YSO configuration consisting of the central star, an optically thick disk, and an optically thin envelope.
\end{abstract}

Key words. radiative transfer - scattering - polarization - circumstellar matter - methods: data analysis methods: numerical

\section{Introduction}

RT simulations provide a well-established basis for the analysis and interpretation of images and polarization maps. The common way is to define a model, to simulate the RT, and to compare the parameters of the escaping radiation with the observed properties of the radiation (e.g., spectral energy distribution [SED], intensity and polarization maps).

The ideal - but in many cases hardly feasible - way is to define a model but not the values of the respective model parameters. Only limits for these values resp. boundary conditions are given. The exact values of the model parameters have to be derived from inverse RT simulations, whereby the observed quantities (i.e., the SED, images and polarization maps) represent the input parameters.

The main problem of inverse RT simulations are nonunique solutions (see, e.g., Thamm et al. 1994). Because of that, this data analysis technique is not widespread. Successful attempts have been undertaken, e.g., by Wang \& Ueno (1989), McCormick (1987), Wilson \& Sen (1986), and Karanjai \& Karanjai (1985a, 1985b). In each case, the models are quite simple and the number of derived parameters is very small (typically $2 \ldots 4$, e.g., the asymmetry factor of scattering particles and the albedo of single scattering).

* e-mail: wolf@tls-tautenburg.de
In this paper, the first inverse three-dimensional Monte-Carlo RT technique for the investigation of polarization and intensity maps is presented. In contrast to direct Monte-Carlo RT simulations, the test-photons are not followed from their origin (e.g., a star or warm dust grains) to the detector but from the detector to their origin. The main idea is to "scan" the model (e.g., a dusty, clumpy shell around a YSO) following test-photons in backward direction.

In Sect. 2.1 a short overview about the RT code is given. The technique of inverting the RT is explained in Sect. 2.2, and the appropriate algorithm is presented in Sect. 2.3. Restrictions on the applicability of the method are discussed in Sect. 2.4. Finally, the method is applied to a YSO configuration in Sect. 3 .

\section{Computational method}

\subsection{Monte-Carlo radiative transfer code}

The method is based on a three-dimensional selfconsistent Monte-Carlo continuum RT code. Because the code has already been described in detail (see, e.g., Wolf et al. 1999 - hereafter WHS99; Wolf \& Henning 2000; Fischer et al. 1994), in this paper only a short introduction into its basic principles is given:

The spatial distribution of the stars and the scattering/absorbing medium (dust grains and/or electrons) is defined inside a convex model space. The radiation energy is partitioned into so-called weighted test-photons 
(particle picture). The energy, intensity, and polarization of a weighted test-photon are described by its wavelength $\lambda_{\mathrm{P}}$ and the Stokes vector $(I, Q, U, V)_{\mathrm{T}}$. The RT for each of these test-photons is subdivided into 3 steps: the emission of a weighted test-photon by the star or a dust grain, its interaction with dust grains or electrons in the stellar environment, and its observation after leaving the model configuration.

\section{Emission from a star or a dust grain:}

The initial Stokes vector of each newly emitted unpolarized test-photon is

$$
\left(\begin{array}{l}
I \\
Q \\
U \\
V
\end{array}\right)_{0}=\left(\begin{array}{l}
1 \\
0 \\
0 \\
0
\end{array}\right)
$$

\section{Scattering and absorption:}

The free path length $l$ of the test-photon can be derived from the optical depth $\tau_{\text {ext }}$ along $l$ :

$\tau_{\text {ext }}=-\ln (1-\zeta)$

where $\zeta$ is a random number uniformly distributed on the interval $[0,1]$.

The modification of the Stokes vector due to the $i$ th interaction can be described with the help of a special $4 \times 4$ Mueller matrix $\hat{S}(\theta)$, where $\theta$ is the scattering angle (see also Bickel \& Bailey 1985; Bohren \& Huffman 1983).

$$
\left(\begin{array}{l}
I \\
Q \\
U \\
V
\end{array}\right)_{i} \propto \hat{S}(\theta)\left(\begin{array}{c}
I \\
Q \\
U \\
V
\end{array}\right)_{i-1}
$$

Here, the scatterers are assumed to be spherical dust grains or electrons. In the case of dust scattering the absorption has to be considered additionally - the weighted test-photon loses energy but will not vanish. The absorption of energy leads to dust heating.

The new direction of the scattered test-photon path is determined by the scattering probability distribution function.

\section{Observation:}

If the test-photon leaves the model space, it will be "observed" by array-detector-like planes being arranged around the model space (see Fig. 1 in WHS99). The Stokes vector has to be projected onto the respective plane.

A more detailed description of the solution of the RT problem in our code, especially the calculation of the spatial dust temperature distribution and the thermal dust re-emission, is outlined in WHS99.

\subsection{Raytracing}

Usually, dust temperature distributions, spectral energy distributions, polarization and intensity maps are the final and only result of RT simulations. Due to the straightforward implementation of the physical processes, Monte-Carlo RT codes - similar to the one described beforehand (see Sect. 2.1) - provide an additional source of information. The paths of the test-photons symbolize the flow of radiative energy inside the model space. Because the number of test-photons needed for RT simulations is extremely large $\left(10^{6} \ldots 10^{9}\right)$, the parameters of the testphotons (Stokes vector, wavelength, last scattering position, recent scattering direction) are stored after escaping the model space only. All information about the paths of the single test-photons inside the model space is lost.

In the method for the analysis of polarization and intensity maps described here, the investigation of the backward - inverse - direction of the test-photon transfer is proposed. Based on polarization/intensity maps derived from Monte-Carlo RT simulations, the paths of all testphotons being accumulated in a selected pixel of the map are raytraced. From the distribution of the test-photon paths the flow of radiative energy into a single pixel can be reconstructed.

This technique can be used as a basis for a more straightforward search for model configurations of spatially resolved dusty envelopes. Instead of varying every parameter of the model configuration, one only has to find out in which pixel(s) the simulated polarization/intensity strongly differs from the observed value. From the distribution of the test-photon paths those regions where the model has to be modified (e.g., modification of the dust density distribution) can be determined. Only those regions with a significant spatial density of test-photon paths contribute to the modification of the intensity/polarization of the test-photons, whereby the other regions have only a negligible influence.

\subsection{Algorithm}

The proposed method is based on the following algorithm:

1. Simulation of the polarization/intensity map;

2. Selection of the region in the map which has to be investigated

- Because the quantity of information arising from the transport of all test-photons is usually very large, it is useful, to restrict the analysis of the polarization/intensity map to a small region, i.e., a single pixel;

3. Repetition of the RT

- The scattering positions of all test-photons which would be collected in the relevant pixel(s) have to be stored;

4. Rebuild the test-photon paths

- Because the test-photon transfer is strongly determined by random processes, many independent test-photons (typically $>10^{4}$, mostly depending on 
the spatial resolution of the map and the optical depth of the configuration) are needed for a meaningful density distribution of test-photon paths.

Finally, if the locations of largest influence on the considered pixels have been found, its model parameters (density distribution, dust grain properties, etc.) have to be changed. Depending on the complexity of the problem (mainly given by the number of free parameters and the structure of the density distribution), different techniques to find the best next choice of model parameters may be applied. In the case of complex models, evolution strategies are most promising.

\subsection{Restrictions on applicability}

This method of data analysis has been primarily developed for "fine-tuning" of model configurations (model geometry, dust/electron density distribution) and detailed investigation of the RT in model configurations. While observations provide two-dimensional maps, this method allows a three-dimensional analysis of the observed objects.

However, the following restrictions of the applicability of the method have to be taken into account:

1. The method does not work unequivocally. This is a fundamental problem of RT simulations being applied to fit real observations (see, e.g., Thamm et al. 1994). It is mainly based on the lack of sufficient model boundary constraints. But, using observations at different wavelengths, the uncertainty in the choice of model parameters can be strongly decreased. This is due to the fact, that the model has to fit the observations for every wavelength taking into account the wavelength dependent optical properties of the dust.

2. The RT problem is nonlocal in nature. Thus - in the general case - same spatial regions and therefore the modifications of its parameters, may contribute equally to the radiation reaching different pixels. One may run into convergence problems because the location of those pixels for which the observed radiation differs significantly from the simulated radiation may change between two consecutive iterative steps. One way out of this problem is to consider the whole radiation escaping from the model space (instead of the radiation reaching a few pixels only) and its change between two consecutive iterative steps. The detailed analysis of such algorithms is beyond the scope of this publication.

Furthermore, in the case of simple model geometries/density distributions - for which we stress the application of this method - the problems described above are mostly of insignificant importance. Additionally, the method may be applied to those models only, in which spatially small regions have to be investigated ("fine-tuning"!). It cannot be used to derive a complete density distribution from the scratch.

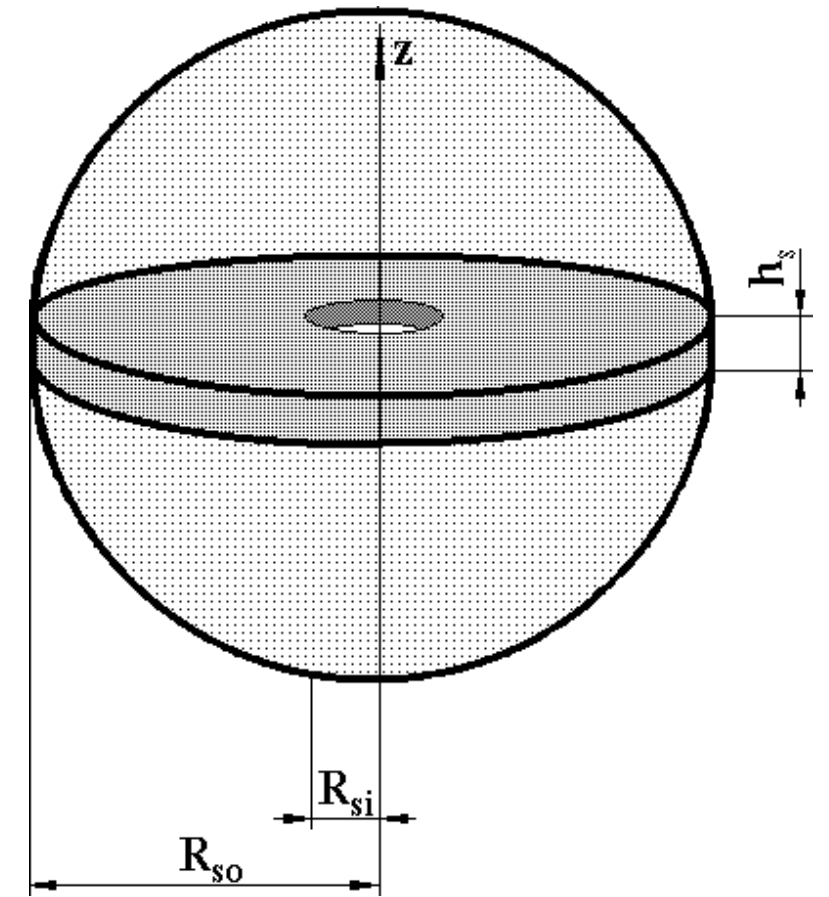

Fig. 1. Model configuration consisting of an optically thick disk, an optically in shell and a dust free regions around the point-like star (see also Sect. 3.1).

The method works best for optically thin $(\tau \leq 10)$ configurations resp. at wavelengths for which the configurations are optically thin. As pointed out in Sect. 3, the distribution of the test-photon paths smears out when the optical depth increases. The higher the optical depth, the less we can learn about inner, embedded structures.

\section{Application}

\subsection{Model configuration}

For the demonstration of the method we define a very simple configuration consisting of a point-like star which is embedded in an optically thin, spherical dust shell. Additionally, the star is surrounded by an optically thick disk with a dust-free region around the star (see Fig. 1; $\left.R_{\mathrm{si}}=12.5 \mathrm{AU}, R_{\mathrm{so}}=500 \mathrm{AU}, h_{\mathrm{s}}=25 \mathrm{AU}\right)$. The dust grains are spherical (radius $a_{\mathrm{Grain}}=0.005 \ldots 0.25 \mu \mathrm{m}$; exponential grain size distribution: $\left.a_{\text {Grain }}(r) \propto r^{-3.5}\right)$ and consist of $62.5 \%$ astronomical silicate and $37.5 \%$ graphite (optical data from Draine \& Lee 1984). The wavelength of the test-photons is $1.25 \mu \mathrm{m}$. The optical depth at this wavelength amounts to 0.2 along the $z$-axis and to 8000 perpendicular to the $z$-axis.

It is the aim of this demonstration to investigate 4 qualitatively different regions - each of them represented by a single pixel - in the polarization map of this object seen edge-on (see Fig. 2): Pixel \#1: region of high linear polarization in the optically thin shell; Pixel \#2: high linear polarization near the optically thick disk; Pixel \#3: lowest linear polarization near the disk (so-called "zero 


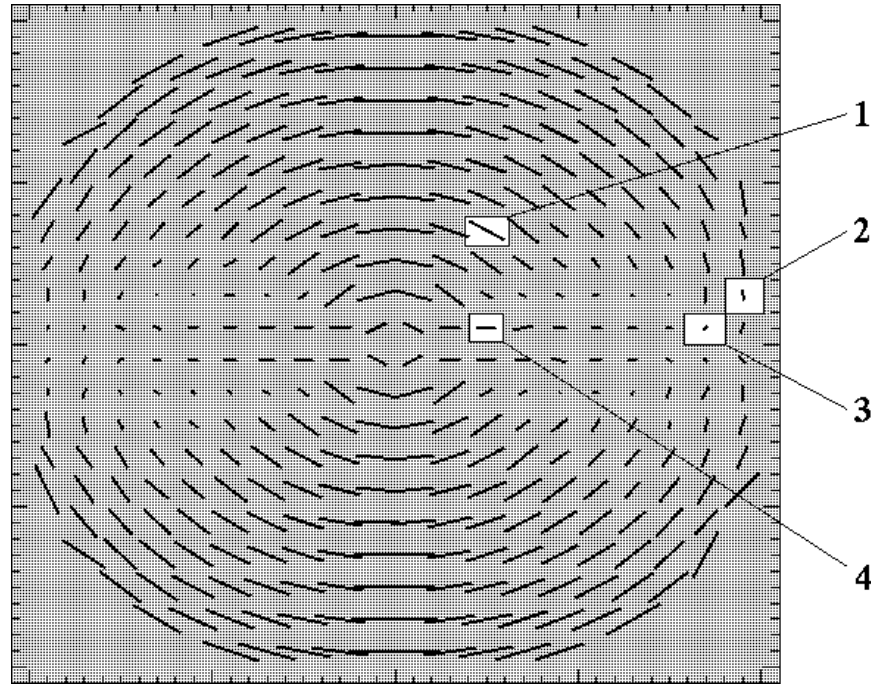

Fig. 2. Polarization map of the configuration shown in Fig. 1 seen edge on. The radiation being detected in four marked pixels is considered (see also Sect. 3.1). The centro-symmetric polarization pattern arises from light scattering in the optically thin shell while scattering by the optically thick disk leads to polarization vectors being oriented parallel to the disk surface.

point of polarization"); Pixel \#4: high linear polarization at the outer boundary of the shell.

\subsection{Test-photon path densities}

Applying the algorithm proposed in Sect.2.1, the testphoton paths are projected onto 3 planes arranged orthogonally around the model space (see Fig. 3). This will allow a three-dimensional reconstruction of the paths. The observer and therefore the polarization map with the marked pixels (Fig. 2) is located opposite to the $y$ - $z$ plane.

In Fig. 4 the qualitative different paths of the testphotons being collected in Pixel \#1 and \#3 are shown. The intensity of the test-photons at each point of their path is taken into account by gray-scaling (dark lines symbolize high intensity). One can see that most of the testphotons which are scattered into Pixel \#1 reach the respective line of sight after only one scattering event. Due to the vertical extension of the optically thick disk, those test-photons which are collected in Pixel \#3 have to be scattered at least once before they reach the respective line of sight. In both cases an additional scattering act is necessary for the test-photon to escape from the shell. The described behaviour is illustrated in the scattering statistics (see Fig. 5)

The symmetry of the test-photon paths in the $y-z$ plane makes clear, that the intensity and polarization of the radiation escaping this optically thin shell is determined equally by the dust density distribution at $x \leq 0$ and $x>0$.

With increasing spatial density of the test-photon paths (dark regions in the projection planes), the influence of (local) variations of the dust parameters, i.e., the

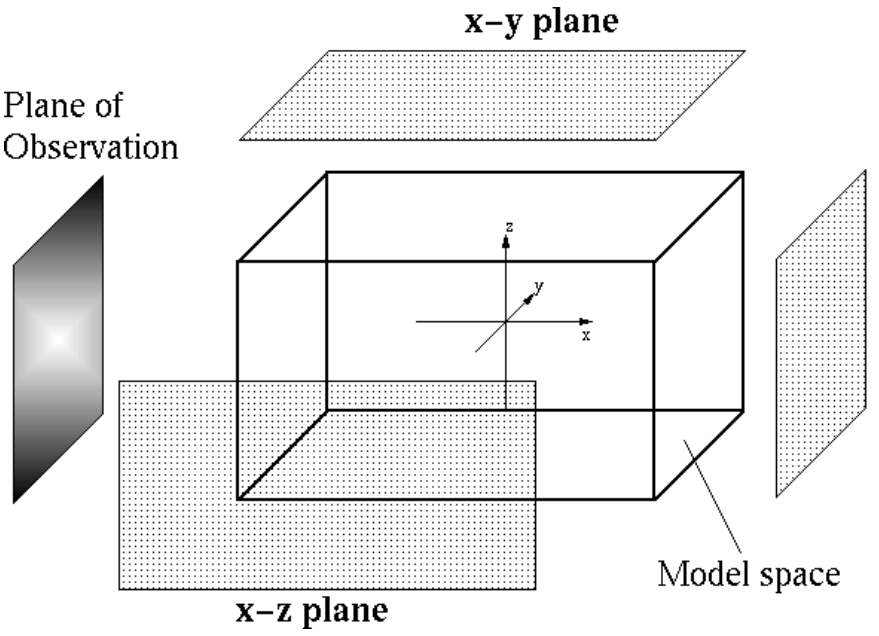

Fig. 3. Arrangement of the planes for the projection of the test-photon paths. The whole model configuration is defined inside the model space. The polarization pattern (see Fig. 2) has been obtained in the plane of observation.

dust density or size distribution as well as the chemical composition, on the observed radiation grows. The chosen way of visualizing the test-photon paths is therefore suitable for the description of the properties and spatial location of such "disturbances". Based on a simple model of an observed object, its individual pecularities can be investigated. As mentioned in Sect. 2.2, this method is practicable for those wavelengths only, for which the objects have a low or moderate optical depth $(\tau \leq 10)$. This is demonstrated by a radiative transfer simulation in an optically thick shell $(\tau=10)$. Due to the increased number of scatterings $(\bar{m}(\tau=10)=11.8, \bar{m}(\tau=0.2)=3.5$, $\bar{m}$... mean number of scatterings of a test-photon before escaping the model space), the resulting test-photon paths are strongly "smeared out" (see Fig. 6; for comparison see Fig. 4, $y$ - $z$ plane for Pixel \#1).

\subsection{Detailed modification of intensity/polarization maps}

In the following it will be shown how the test-photon path densities derived in Sect. 3.2 can be used for a detailed modification of the polarization map, resp. the intensity map. For this reason it is assumed that the simulated polarization map shown in Fig. 2 almost perfectly agrees with an observed polarization map of a YSO configuration with a geometry as shown in Fig. 1. Furthermore, it is assumed that the only remarkable deviation between the simulated and the observed polarization/intensity map appears in the region represented by Pixel \#1. To modify the intensity and polarization measured in this region, the quantity

$\zeta(r)=\frac{I_{\mathrm{tp}}(r) \cdot \rho_{\mathrm{tp}}(r)}{\max \left[I_{\mathrm{tp}}(r) \cdot \rho_{\mathrm{tp}}(r)\right]}$

is defined. It combines the mean density of test-photon paths $\rho_{\text {tp }}(r)$ (of all photons collected in Pixel \#1) with the 


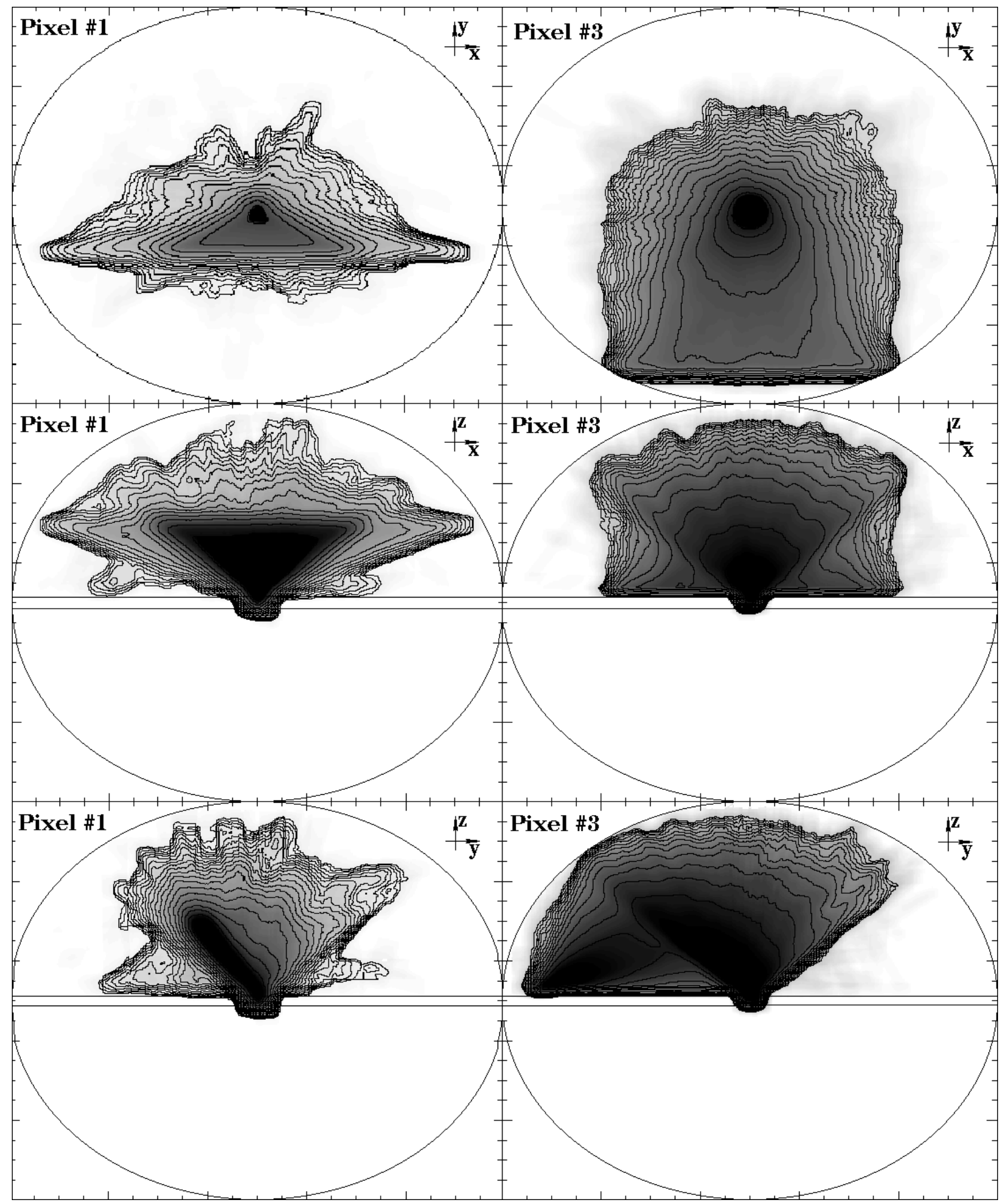

Fig. 4. Projections with overlayed contour plots of the spatial density of the test-photon paths for Pixel \#1 and \#3 onto the 3 orthogonally arranged projection planes (see Fig. 3). Dark gray regions mark a high spatial density of test-photon paths. In the upper right edge of each plot the respective plane is denoted. The circles mark the outer boundary of the shell. The parallel lines in the lower 4 plots mark the location of the disk. 


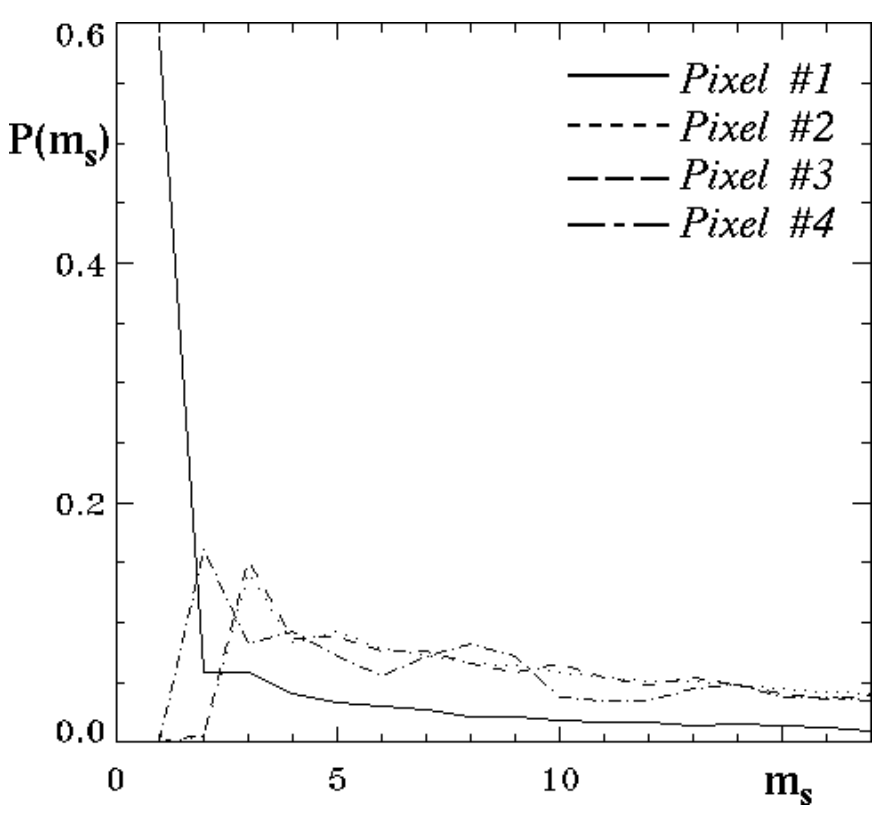

Fig. 5. Scattering statistics for the test-photons being detected in the four pixels marked in Fig. 2. $\left(m_{\mathrm{s}} \ldots\right.$ number of scatterings; $P\left(m_{\mathrm{s}}\right) \ldots$ probability for test-photon to leave the model space after $m_{\mathrm{s}}$ scattering acts.) Only test-photons being collected in Pixel \#1 can reach the resp. line of sight directly.

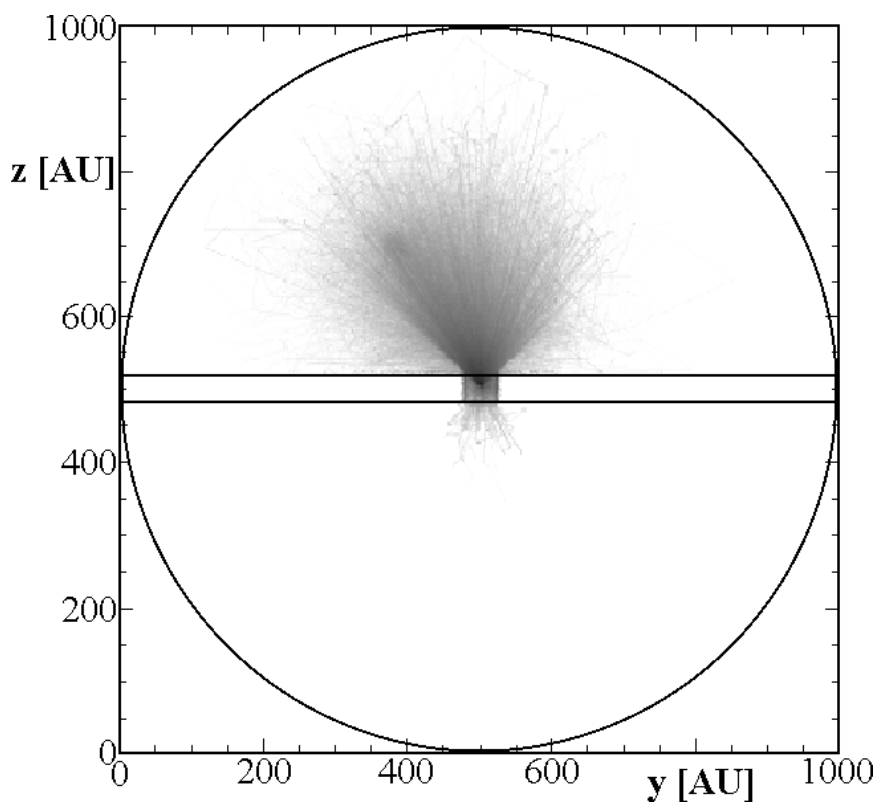

Fig. 6. $y-z$ plane projection of the paths of all test-photons being collected in Pixel \#1. The shell is optically thick $(\tau=$ 10). The circle and the 2 parallel lines mark the outer boundary of the shell resp. the location of the disk. The distribution of the paths is smeared out. For comparison see Fig. 4, $y$ - $z$ plane for Pixel \#1.

corresponding mean intensity $I_{\mathrm{tp}}(r)$ of the test-photons at the position $r$ in the model space. Thus, the quantity $\zeta(r)$ provides a measure for the influence of the dust number density at the position $r$. Modifications of the dust density distribution according to $\zeta_{\text {tp }}(r)$ result in a specific modification of the observable quantities mapped in

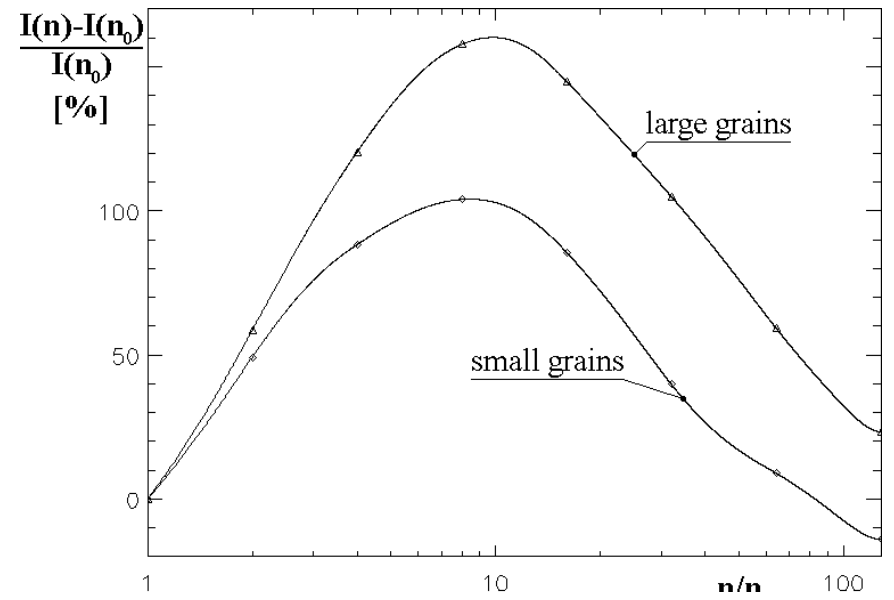

Fig. 7. Relative change of the intensity $I$ measured in Pixel \#1 due to the increase of the dust density distribution in the region where $\zeta(r)>90 \%$. See Sect. 3.3 for detailed explanations.

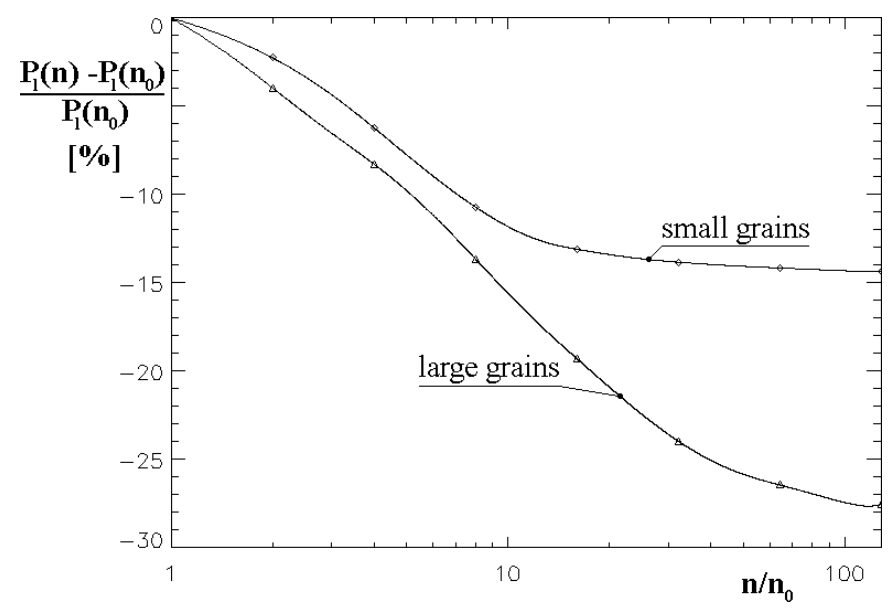

Fig. 8. Relative change of the linear polarization $P_{1}$ measured in Pixel \#1 due to the increase of the dust density distribution in the region where $\zeta(r)>90 \%$. See Sect. 3.3 for detailed explanations.

Pixel \#1. For demonstration, the dust number density was increased in those regions where $\zeta(r)>90 \%$. The resulting relative changes of the intensity and polarization are shown in Figs. 7 and 8 for two different grain size distributions: (1) a small grain population with grain radii $a_{\text {Grain }}=0.005 \ldots 0.25 \mu \mathrm{m}$ and (2) a large grain population $\left(a_{\text {Grain }}=0.1 \ldots 1.0 \mu \mathrm{m}\right)$. In both cases the optical depth of the disk and the shell of the unmodified density distribution was the same. While the increased number of scatterings - caused by the increased density - results in a decrease of the linear polarization degree $P_{1}$, the intensity measured in Pixel \#1 reaches its maximum at $n / n_{0} \approx 10$ (here, $n_{0}(n)$ is the original (increased) dust density in the region where $\zeta(r)>90 \%)$. This maximum marks the transition from the optically thin to the opticall thick regime along the line of sight of this pixel.

As described in Sect. 2.4, the RT is nonlocal in nature and the modification of the dust density in a selected region may influence the radiation reaching different pixels. 
Therefore, the influence of the modification of the dust density distribution on all other pixels (except Pixel \#1) was considered. It was found that the relative change of the intensity and polarization in all other pixels was always below $10 \%$ of the change of these observable quantities in Pixels \#1.

\section{Conclusions}

A method for detailed investigations of polarization and intensity maps is proposed. Based on Monte-Carlo RT simulations the paths of all test-photons being scattered into selected pixels of the maps - representing the regions to be investigated - are raytraced. This way, the inverse investigation of the RT is provided.

From the spatial density distribution of the testphoton paths it is possible to derive those locations where the scattering medium has the most important influence on the observed radiation. This is the base for a straightforward, purposeful modification of models to fit observed polarization and intensity maps. The method is meant to be applied to the investigation of polarization and intensity maps of YSOs, UCHIIs, and AGNs. It is limited to those wavelengths at which the optical depth is lower than approximately 10 .

For the demonstration of this method the RT in a simple model of a YSO is considered. It is shown, that a threedimensional reconstruction of the test-photon paths and therefore a three-dimensional investigation of the considered objects is possible.
Finally, it was demonstrated how this method can be used for a detailed modification of a intensity map and the corresponding polarization map of a YSO configuration.

Acknowledgements. I thank O. Fischer for many helpful comments during the development of the presented method. I wish to thank Th. Henning, B. Stecklum, and the referee for their careful reading of the manuscript. This research was supported by the DFG grant Ste 605/10 within the program "Physics of star formation".

\section{References}

Bickel, W. S., \& Bailey, W. M. 1985, Am. J. Phys., 53(5), 468

Bohren, C. F., \& Huffman, D. R. 1983, Absorption and scattering of light by small particles (John Wiley \& Sons, New York)

Draine, B. T., \& Lee, H. M. 1984, ApJ, 285, 89

Fischer, O., Henning, Th., \& Yorke, H. W. 1994, A\&A, 284, 187

Karanjai, S., \& Karanjai, M. 1985a, Ap\&SS, 117, 151

Karanjai, S., \& Karanjai, M. 1985b, Ap\&SS, 117, 387

McCormick, N. J. 1987, Ap\&SS, 129, 331

Thamm, E., Steinacker, J., \& Henning, Th. 1994, A\&A, 287, 493

Wang, A. P., \& Ueno, S. 1989, Ap\&SS, 155, 105

Wilson, S. J., \& Sen, K. K. 1986, Ap\&SS, 119, 93

Wolf, S., \& Henning, Th. 2000, Comp. Phys. Comm., 132, 166

Wolf, S., Henning, Th., \& Stecklum, B. 1999, A\&A, 349, 839, [WHS99] 